\title{
Radical--Promoted Stone--Wales Rearrangements
}

\author{
Roger W. Alder,* and Jeremy N. Harvey \\ Supplementary Material
}

SCF energies, zero point corrections, imaginary frequencies, and Cartesian coordinates for intermediates and transition states.

\begin{abstract}
Hydrogen atom-promoted scrambling of C-3 and C-4 in Hexatriene

C $-1.3908-0.96091 .9238$

H $-0.8533-1.90701 .8971$

H -2.3917 -0.98212 .3468$

C $-0.8477 \quad 0.1650 \quad 1.4616$

H - $1.4219 \quad 1.0918 \quad 1.5007$

$\begin{array}{llll}\text { C } & 0.5350 & 0.2734 & 0.8547\end{array}$

H $1.1376 \quad 0.9597 \quad 1.4745$

H $1.0313-0.70420 .8920$

C $0.50040 .7823-0.5604$

H $0.09161 .7809-0.7183$

C $0.92590 .0585-1.6664$

H $1.3208-0.9427-1.4845$

C $0.89160 .5077-2.9764$

H $0.50961 .4950-3.2233$

H $1.2431-0.1083-3.7973$
\end{abstract} hexatriene (Scheme 3 and Figure 1)

\section{Hydrogen atom adduct to $\mathrm{C} 3$ of}

SCF energy -233.969586803 a.u.

Zero-point correction 0.12862 a.u.

First transition state

SCF energy -233.939284886 a.u. Zero-point correction 0.12765 a.u. Imaginary frequency $-529.36 \mathrm{~cm}^{-1}$

H -2.2466 $0.6493-0.0552$

C - $1.18840 .9029-0.1123$

C $-0.2384-0.0572 \quad 0.5171$

C $-0.41840 .2636-1.2221$

H - $1.00201 .9621 \quad 0.0657$

H -0.6168 $-1.0478 \quad 0.7504$

H $0.47770 .7858-1.5460$

C $-0.9090-0.7718-2.0392$
H $-0.2987-1.1937-2.8303$

H - $1.8630-1.2482-1.8315$

C 1.01620 .33861 .1178

H $1.3381 \quad 1.3645 \quad 0.9281$

C $1.8003-0.45621 .8740$

H $2.7329-0.09712 .2976$

H $1.5243-1.48492 .0943$

First intermediate

SCF energy -233.941899763 a.u.

Zero-point correction 0.12845 a.u.

H $-2.32590 .7112-0.1202$

C $-1.27420 .9794-0.1845$

C $-0.2646-0.0345 \quad 0.2751$

C $-0.45040 .2999-1.2547$

$\mathrm{H}-1.0705 \quad 2.0281 \quad 0.0205$

H $-0.6529-1.0075 \quad 0.5674$

H $0.36960 .8886-1.6608$

C $-1.0186-0.6897-2.1508$

H $-0.4936-0.9944-3.0492$

H - $1.9169-1.2351-1.8767$

$\begin{array}{llll}\text { C } & 0.9822 & 0.3767 & 0.9420\end{array}$

H 1.35581 .36840 .6795

C $1.6683-0.36751 .8163$

H $2.5872-0.00952 .2713$

H $1.3320-1.36012 .1084$

Second (rate-limiting) transition state

SCF energy -233.92430527967 a.u. Zero-point correction 0.12680 a.u. Imaginary frequency $-556.36 \mathrm{~cm}^{-1}$

$\mathrm{H}-2.2854 \quad 0.7207 \quad 0.0627$

C - $1.24010 .9952 \quad 0.1182$

C $-0.1960-0.05320 .2919$

C $-0.2007 \quad 0.0427-1.2113$
H - $0.97662 .0468 \quad 0.1320$

H $-0.5902-1.0087 \quad 0.6484$

H $0.42680 .8243-1.6335$

C $-0.8318-0.8441-2.0582$

$\mathrm{H}-0.8308-0.6912-3.1325$

H - $1.3967-1.6898-1.6749$

C 1.06790 .32760 .9942

H $1.5118 \quad 1.2763 \quad 0.6870$

C $1.6619-0.40001 .9407$

H $2.5824-0.07492 .4178$

H $1.2495-1.35002 .2747$

\section{Second intermediate}

SCF energy -233.937307420 a.u.

Zero-point correction 0.12743 a.u.

C $1.1780 \quad 0.7001-0.9341$

H $1.95401 .3172-0.4934$

H $1.30790 .3635-1.9580$

C $-0.1477 \quad 0.5530-0.2535$

H $-0.81381 .3672-0.6024$

C $-0.8625-0.7545-0.5638$

H $-1.9200-0.7623-0.2995$

C $-0.0258 \quad 0.7058 \quad 1.2531$

H $0.6181-0.02761 .7388$

C $-0.3069-1.8562-1.0699$

H $0.7475-1.9017-1.3305$

H - $0.8911-2.7571-1.2373$

C $-0.6478 \quad 1.6343 \quad 1.9797$

$\mathrm{H}-0.52961 .69023 .0587$

H -1.2996 2.3766 1.5223 
Methyl radical-promoted scrambling of C-3 and C-4 in hexatriene (Scheme 3 and Figure 1)

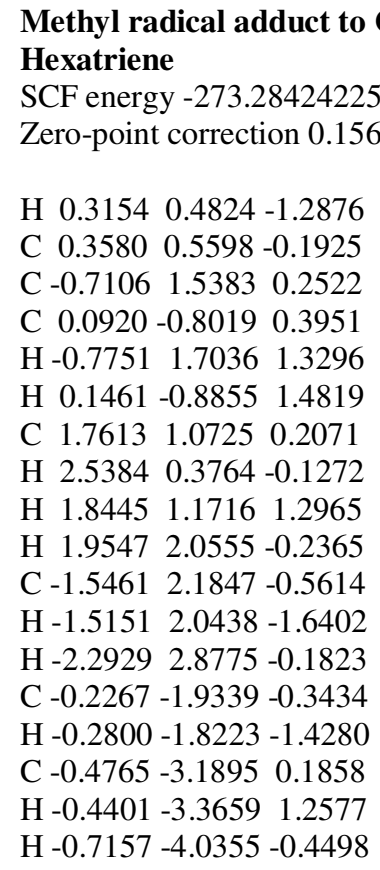

First transition state

SCF energy -273.25540196976 a.u. Zero-point correction 0.15594 a.u. Imaginary frequency $-503.89 \mathrm{~cm}^{-1}$

H $0.1577 \quad 1.0638-1.5754$

C $0.31540 .8748-0.5111$

$\begin{array}{llll}\text { C } & -0.9669 & 0.7448 & 0.2582\end{array}$

C $0.1676-0.5613-0.1292$

$\mathrm{H}-1.8567 \quad 0.5811-0.3440$

H $0.6785-0.87820 .7764$

C $1.4740 \quad 1.6744 \quad 0.0572$

H $2.39181 .4694-0.5057$

H 1.66621 .41901 .1045

H 1.27002 .75010 .0060

C $-0.3091-1.5938-1.0229$

H - $0.7451-1.2519-1.9636$

C - 1.16421 .09391 .6087

H $-0.3461 \quad 1.39732 .2532$

C $-0.2581-2.9189-0.7774$

H $0.1663-3.31130 .1439$

$\mathrm{H}-0.6326-3.6442-1.4930$

H $-2.1405 \quad 0.97712 .0665$

First intermediate

SCF energy -273.257589388 a.u.

Zero-point correction 0.15670 a.u.

H $0.1757 \quad 1.0366-1.5851$

C $0.32850 .8674-0.5188$

$\begin{array}{llll}C & -0.9597 & 0.6558 & 0.2571\end{array}$

C $0.0347-0.5339-0.0563$
H - $1.8583 \quad 0.6173-0.3559$

$\begin{array}{llll}\text { H } & 0.5951 & -0.8733 & 0.8132\end{array}$

C 1.49341 .64390 .0592

H $2.4167 \quad 1.4145-0.4860$

H 1.66501 .39741 .1122

H $1.31932 .7247-0.0059$

C $-0.3879-1.5926-0.9868$

H $-0.9139-1.2557-1.8821$

C $-1.1702 \quad 1.1045 \quad 1.6200$

H $-0.3418 \quad 1.2907 \quad 2.2947$

C $-0.1802-2.9022-0.8081$

$\begin{array}{llll}\mathrm{H} & 0.3395 & -3.2833 & 0.0685\end{array}$

$\mathrm{H}-0.5208-3.6373-1.5315$

H -2.1728 1.15592 .0297

Second transition state (ratelimiting)

SCF energy -273.24310591542 a.u.

Zero-point correction 0.15527 a.u.

Imaginary frequency $-534.45 \mathrm{~cm}^{-1}$

H $0.13761 .0796-1.6463$

C $0.44910 .8466-0.6313$

C $-1.1259 \quad 0.5024 \quad 0.4154$

C $-0.0410-0.4370-0.0474$

H - $1.92430 .6876-0.2994$

$\begin{array}{llll}\text { H } & 0.5749 & -0.7971 & 0.7821\end{array}$

C $1.55291 .6469-0.0160$

H $2.54111 .2801-0.3373$

H 1.52361 .58981 .0782

H $1.49012 .7049-0.2950$

C $-0.4280-1.5422-0.9760$

H - $1.0449-1.2506-1.8279$

C $-1.2477 \quad 1.00001 .6986$

H $-0.5381 \quad 0.74382 .4803$

C $-0.0730-2.8193-0.8297$

H $0.5406-3.14980 .0061$

H $-0.3822-3.5835-1.5377$

H $-2.03281 .7051 \quad 1.9525$

\section{Second intermediate}

SCF energy -273.257756607 a.u.

Zero-point correction 0.15552 a.u.

$\begin{array}{llll}\text { H } & 0.7425 & 0.7133 & -1.9527\end{array}$

C $0.76930 .7553-0.8670$

$\begin{array}{llll}C & -1.2337 & 0.4523 & 0.5637\end{array}$

C $-0.0416-0.2457-0.0788$

H $-1.9841 \quad 0.8287-0.1325$

H $0.5799-0.65010 .7369$

C $1.64651 .7494-0.1831$

H $2.6119 \quad 1.30720 .1222$

H 1.17632 .13260 .7329

H $1.87762 .6026-0.8306$

C $-0.5014-1.3934-0.9504$

H - $1.1228-1.1139-1.8034$

C -1.3856 0.65191 .8739

H -0.65590 .28862 .5952$

C - $0.2029-2.6763-0.7491$

H $0.4132-2.9948 \quad 0.0898$
H -0.5619 -3.4584 -1.4131

H -2.2474 1.17782 .2766

Rearrangement of

bifluorenylidene to

dibenzo[g,p]chrysene (Scheme 4)

Hydrogen atom adduct to bifluorenylidene

SCF energy -1001.01099153568 a.u.

Zero-point correction 0.34507 a.u.

C $0.0867-0.73052 .7791$

C -0.0260 0.73042 .9098

C $-0.5221 \quad 1.2547 \quad 1.6988$

C - $0.3412-1.10231 .4885$

C $1.33760 .5703-2.3919$

C $-0.0047 \quad 0.4811-2.9765$

C $-0.93020 .3104-1.8969$

C - $0.2012 \quad 0.2916-0.6557$

C $1.19920 .4522-0.9729$

C $-0.8118 \quad 0.1265 \quad 0.7073$

C $-0.3175-2.4325 \quad 1.0851$

C $0.1355-3.40251 .9871$

C $0.5554-3.03863 .2723$

C $0.5354-1.70163 .6785$

C $0.2814 \quad 1.58423 .9727$

C $0.09312 .9593 \quad 3.8090$

C - $0.3940 \quad 3.47642 .6023$

C $-0.7057 \quad 2.6232 \quad 1.5370$

C $-0.4556 \quad 0.5331-4.2889$

C $-1.8316 \quad 0.4152-4.5421$

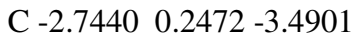

C - $2.3041 \quad 0.1937-2.1662$

C $2.3377 \quad 0.5027-0.1528$

C $3.59140 .6691-0.7455$

C $3.71990 .7844-2.1369$

C $2.59090 .7352-2.9686$

H $4.70540 .9131-2.5771$

H $4.47930 .7096-0.1200$

H -2.1940 $0.4545-5.5660$

H -3.8049 $0.1578-3.7089$

H $0.2413 \quad 0.6627-5.1136$

H $0.3313 \quad 3.63554 .6261$

H -0.5284 4.54922 .4908

H $0.9052-3.80433 .9601$

H $0.1637-4.44671 .6863$

H $2.70190 .8252-4.0467$

H $0.6643 \quad 1.18994 .9107$

H $0.8675-1.42554 .6762$

H - 1.90200 .05430 .5789

H $-0.6371 \quad-2.7141 \quad 0.0847$

H -3.0217 $0.0631-1.3595$

H - $1.0773 \quad 3.0253 \quad 0.5975$

H $2.2487 \quad 0.41480 .9252$ 
First (rate-limiting) transition state SCF energy -1000.94111084873 a.u. Zero-point correction 0.34296 a.u. Imaginary frequency $-531.08 \mathrm{~cm}^{-1}$

C $2.6809-0.8678-0.0357$

C $2.38620 .3988-0.7119$

C $1.03280 .7420-0.4850$

C $1.5060-1.32730 .5904$

$\begin{array}{llll}C-2.2941 & 1.0306 & 0.2880\end{array}$

C $-2.0963-0.1371-0.5683$

C $-0.9814-0.9930-0.1712$

C $-0.5872-0.2006 \quad 1.4590$

$\begin{array}{llll}\mathrm{C}-1.5217 & 0.9281 & 1.4703\end{array}$

C $0.3678-0.36440 .3240$

C $1.5393-2.4797 \quad 1.3740$

C $2.7410-3.18791 .4992$

C $3.9000-2.74260 .8545$

C $3.8786-1.57470 .0879$

C $3.22731 .2224-1.4614$

C $2.71572 .4083-1.9919$

C $1.38012 .7557-1.7692$

C $0.5321 \quad 1.9285-1.0218$

C $-3.0268-0.5975-1.4954$

C $-2.9698-1.9029-1.9919$

C - $1.9833-2.7846-1.5103$

C - $1.0144-2.3547-0.6219$

C - $1.6847 \quad 1.85832 .4994$

$\begin{array}{lll}\text { C }-2.5808 & 2.9184 & 2.3328\end{array}$

C -3.3136 3.04631 .1459

$\begin{array}{llll}\text { C }-3.1789 & 2.1031 & 0.1249\end{array}$

H -3.9991 3.88051 .0211

H -2.7009 $3.6523 \quad 3.1253$

H -3.7102 -2.2468 -2.7083

H $-1.9582-3.8122-1.8643$

H $-3.85520 .0484-1.7768$

H $3.35703 .0623-2.5773$

H $0.98813 .6824-2.1806$

H $4.8254-3.30310 .9594$

H $2.7710-4.09052 .1038$

H -3.7658 2.2002 -0.7852

H $4.26560 .9465-1.6283$

H $4.7849-1.2177-0.3946$

H -0.4101 -0.79302 .3523$

H $0.6479-2.83861 .8824$

H - $0.2178-3.0306-0.3366$

H - $0.49582 .2283-0.8681$

H -1.1024 1.77003 .4133

\section{First intermediate}

SCF energy -1000.998991872 a.u.

Zero-point correction 0.34454 a.u.

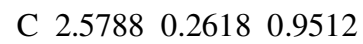

C $2.6552-0.6795-0.1768$

C $1.3743-0.8015-0.7491$

$\begin{array}{llll}C & 1.2510 & 0.7158 & 1.0698\end{array}$

$\begin{array}{lll}C-2.4961 & 0.6589 & -0.7306\end{array}$

C $-2.1594-0.4233 \quad 0.2176$

C $-0.8143-0.69440 .5585$
C $-0.0955 \quad 1.1870-0.9618$

C - $1.4297 \quad 1.4433-1.2943$

C $0.35510 .1036-0.0261$

C $0.8927 \quad 1.61952 .0637$

C 1.87542 .06762 .9544

C 3.19681 .61772 .8409

C 3.55920 .71281 .8392

C $3.7308-1.4042-0.6972$

C $3.5101-2.2472-1.7900$

C $2.2348-2.3643-2.3568$

C $1.1568-1.6367-1.8390$

C $-3.1637-1.21890 .8145$

C $-2.8585-2.23801 .7062$

C - $1.5241-2.49862 .0350$

C $-0.5207-1.72721 .4605$

C $-1.75182 .4942-2.2061$

C -3.0600 $2.7646-2.5547$

C $-4.10021 .9938-2.0051$

C -3.8101 $0.9628-1.1103$

H $-5.13292 .1976-2.2743$

H -3.2851 $3.5684-3.2509$

H -3.6586 -2.8284 2.1454

$\mathrm{H}-1.2705-3.29352 .7313$

H -4.2065 - $1.0368 \quad 0.5781$

H $4.3372-2.8184-2.2039$

H $2.0803-3.0261-3.2052$

H 3.94871 .97533 .5398

H 1.61042 .76993 .7405

$\begin{array}{lll}\mathrm{H}-4.6381 & 0.3892 & -0.7070\end{array}$

H $4.7233-1.3183-0.2613$

$\begin{array}{llll}\text { H } & 4.5868 & 0.3667 & 1.7571\end{array}$

H 0.6887 1.7966-1.4032

H -0.1335 1.96762 .1504

H $0.5185-1.92261 .7115$

H $0.1663-1.7263-2.2779$

H -0.9363 $3.0802-2.6237$

Second transition state

SCF energy -1000.9642350859 a.u.

Zero-point correction 0.34320 a.u.

Imaginary frequency $-389.84 \mathrm{~cm}^{-1}$

C $2.7193-1.29431 .1168$

C $2.2125 \quad 0.9778-1.2902$

C $1.04481 .0078-0.5168$

C $1.4696-1.37500 .5106$

C $-2.4683 \quad 1.18262 .0040$

C $-1.6565-0.5555-1.4581$

C $-0.6695-0.9929-0.5017$

C $-0.6425-0.1529 \quad 1.0129$

C $-1.3448 \quad 1.1115 \quad 1.1595$

C $0.5425-0.25930 .0986$

C $0.8511-2.63060 .2882$

C $1.5021-3.80860 .6835$

C $2.7584-3.72151 .2828$

C $3.3661-2.47641 .4978$

C $2.69402 .1251-1.9153$

C $2.00183 .3279-1.7621$

C $0.84093 .3691-0.9955$

C $0.32882 .2208-0.3603$
C - $2.4753-1.4914-2.0477$

C $-2.3317-2.8796-1.7865$

C - $1.2901-3.3403-0.9742$

C $-0.4255-2.4290-0.3766$

C $-0.9065 \quad 2.2728 \quad 0.4667$

C - $1.6547 \quad 3.45310 .6264$

C -2.7764 $3.5047 \quad 1.4504$

C -3.1823 2.36462 .1532

H -3.3310 4.43431 .5462

H -1.3630 4.35660 .1019

$\mathrm{H}-3.0035$-3.5880 -2.2624

$\mathrm{H}-1.1222-4.4090-0.8588$

H -3.2448 -1.1610 -2.7414

H $2.3638 \quad 4.2353-2.2385$

H $0.33064 .3200-0.8885$

H $3.2731-4.6297 \quad 1.5863$

H $1.0377-4.77790 .5205$

H -4.0530 2.39872 .8026

H $3.59762 .0794-2.5172$

H $4.3454-2.42751 .9666$

H - $0.7251-0.87181 .8217$

H $3.1876-0.32911 .2905$

H $2.73990 .0356-1.4052$

H -2.7798 0.28442 .5323

H - $1.78310 .5027-1.6622$

Second intermediate

SCF energy - 1000.965406635 a.u.

Zero-point correction 0.34392 a.u.

C $2.8126-1.33361 .1543$

C $2.29090 .9528-1.2239$

C $1.12310 .9798-0.4480$

C $1.5463-1.40260 .5817$

C $-2.4150 \quad 1.1466 \quad 2.0345$

C $-1.5976-0.5746-1.3773$

C $-0.6475-1.0020-0.3570$

C $-0.5994-0.2111 \quad 1.0365$

C $-1.2932 \quad 1.07891 .1943$

$\begin{array}{llll}C & 0.6341 & -0.2781 & 0.1784\end{array}$

C $0.9076-2.65440 .3885$

C $1.5529-3.83550 .7846$

C $2.8242-3.75891 .3529$

C $3.4533-2.5197 \quad 1.5351$

C $2.75832 .0958-1.8657$

C $2.04973 .2920-1.7351$

C $0.88963 .3311-0.9676$

C $0.39712 .1898-0.3063$

C - $2.3671-1.5136-2.0149$

C - $2.2156-2.9064-1.7597$

C - $1.1959-3.3620-0.9138$

C - $0.3729-2.4521-0.2645$

$\begin{array}{llll}C-0.8325 & 2.2430 & 0.5301\end{array}$

C - $1.5548 \quad 3.4358 \quad 0.7244$

C - $2.6780 \quad 3.4871 \quad 1.5452$

C -3.1111 2.3373 2.2122

$\mathrm{H}-3.21124 .42601 .6681$

$\mathrm{H}-1.2383 \quad 4.3495 \quad 0.2334$

H -2.8512 -3.6194 -2.2762

H - 1.0053 -4.4291 - 0.8218 
H -3.1088 -1.1888 -2.7406

H $2.39674 .1941-2.2320$

H $0.3618 \quad 4.2748-0.8858$

H $3.3328-4.6701 \quad 1.6574$

H $1.0713-4.8003 \quad 0.6462$

H -3.9837 2.36982 .8590

H $3.66152 .0511-2.4683$

H $4.4439-2.47681 .9798$

H $-0.6438-0.87701 .8952$

H $3.2985-0.37321 .3041$

H $2.82720 .0145-1.3284$

$\begin{array}{lll}\mathrm{H}-2.7427 & 0.2415 & 2.5407\end{array}$

H - $1.73680 .4839-1.5706$

Third transition state

SCF energy -1000.96300873334 a.u.

Zero-point correction 0.34366 a.u.

Imaginary frequency $-377.68 \mathrm{~cm}^{-1}$

C $2.7477-1.3267 \quad 1.1109$

C $2.22660 .9661-1.2338$

C $1.06101 .0106-0.4472$

C $1.4574-1.36310 .5846$

C - $2.5019 \quad 1.20841 .9828$

C $-1.8004-0.5653-1.3121$

C $-0.8666-0.9892-0.2952$

C $-0.6811-0.17121 .0041$

C $-1.3767 \quad 1.13551 .1520$

C $0.5938-0.19860 .2364$

C $0.7974-2.60260 .3921$

C $1.4343-3.79580 .7595$

C $2.7236-3.7508 \quad 1.2914$

C $3.3808-2.52531 .4617$

C $2.65862 .0782-1.9470$

C $1.92073 .2635-1.8880$

C $0.7683 \quad 3.3242-1.1083$

C $0.3150 \quad 2.2191-0.3650$

C $-2.4225-1.5006-2.1078$

C - $2.1509-2.8847-1.9694$

C $-1.1612-3.3149-1.0782$

C $-0.4791-2.4019-0.2815$

C -0.89802 .29360 .4948$

C - 1.59673 .49940 .7078

$\begin{array}{llll}C & -2.7225 & 3.5589 & 1.5247\end{array}$

C -3.1831 2.40792 .1688

$\mathrm{H}-3.23654 .50651 .6623$

H - $1.2557 \quad 4.4153 \quad 0.2384$

H -2.6758 -3.6051 -2.5899

$\mathrm{H}-0.8710-4.3633-1.0588$

H -3.1495 -1.1712 -2.8463

H $2.23774 .1378-2.4502$

H 0.2124 4.2551 -1.0877

H $3.2224-4.67491 .5721$

H $0.9327-4.7508 \quad 0.6240$

H -4.0599 2.4462 2.8094

H $3.55782 .0183-2.5545$

H $4.3860-2.50141 .8741$

H $-0.7120-0.78521 .9051$

H $3.2538-0.37601 .2558$

H $2.78310 .0357-1.2897$
H -2.8436 0.30392 .4811

\section{Hydrogen atom adduct to dibenzo[g,p]chrysene}

SCF energy -1001.00806252 a.u.

Zero-point correction 0.34645 a.u.

C $2.8431-1.35790 .9789$

C $2.57141 .1763-0.9387$

C $1.31211 .1687-0.2707$

C $1.5182-1.27010 .5101$

C $-2.5825 \quad 1.26281 .5622$

C $-2.5341-1.0161-0.7752$

C - $1.2933-1.1698-0.1579$

C $-0.6449-0.1107 \quad 0.7576$

C $-1.3596 \quad 1.21620 .8794$

C $0.8036-0.00650 .3489$

C $0.7951-2.47250 .2527$

C $1.4156-3.70460 .5016$

C $2.7265-3.77080 .9730$

C $3.4433-2.59321 .2081$

C $3.04062 .3011-1.5939$

C $2.26173 .4666-1.6262$

C $1.01803 .4820-0.9969$

C $0.51942 .3692-0.3063$

C $-3.0690-2.0406-1.5624$

C $-2.3578-3.2276-1.7369$

C $-1.1080-3.3819-1.1369$

C $-0.5584-2.3651-0.3415$

C $-0.7882 \quad 2.40650 .3827$

C $-1.4853 \quad 3.6147 \quad 0.5937$

C $-2.70803 .6456 \quad 1.2543$

C -3.2661 $2.4617 \quad 1.7453$

H -3.2179 4.59451 .3998

H - 1.05664 .55210 .2562

H $-2.7632-4.0252-2.3541$

H $-0.5394-4.2900-1.3158$

H $-4.0352-1.9041-2.0413$

H $2.6125 \quad 4.3498-2.1529$

H $0.41964 .3843-1.0639$

H $3.1838-4.73761 .1660$

$\begin{array}{llll}\text { H } & 0.8635 & -4.6250 & 0.3337\end{array}$

H -4.21432 .47562 .2760$

H $4.00082 .2694-2.1024$

H $4.4603-2.63751 .5890$

H -0.6594 -0.5644 1.7648

H $3.3830-0.44361 .2063$

H $3.15270 .2621-0.9663$

H $-2.99690 .3378 \quad 1.9574$

H -3.0868 -0.0914 -0.6466

$\mathrm{C}$ (carbene)-C( $\left.\mathrm{sp}^{3}\right)$ intermediate in non-catalysed rearrangement of bifluorenylidene

SCF energy -1000.313796398 a.u.

Zero-point correction 0.33248 a.u.

C 2.50440 .31380 .9113

C $2.4424-0.4814-0.3157$

C $1.0976-0.5776-0.7349$
C $1.1963 \quad 0.6976 \quad 1.2674$

C - $2.48780 .5028-0.8633$

C - $2.2666-0.4665 \quad 0.2318$

C $-0.9739-0.6056 \quad 0.7811$

C $-0.0752 \quad 1.3443-0.7248$

C - $1.4126 \quad 1.3487-1.2852$

$\begin{array}{llll}C & 0.1763 & 0.2207 & 0.2320\end{array}$

C $0.9661 \quad 1.45402 .4123$

C $2.0566 \quad 1.82473 .2064$

C 3.35951 .45082 .8510

C 3.59370 .69481 .7009

C $3.4432-1.1347-1.0392$

C $3.0888-1.8802-2.1665$

C $1.7510-1.9783-2.5701$

C $0.7437-1.3201-1.8577$

C $-3.3095-1.25890 .7545$

C -3.0795 -2.1704 1.7780

C - $1.7928-2.31162 .3091$

C $-0.7545-1.5307 \quad 1.8104$

C - $1.63962 .2960-2.3121$

C -2.8713 $2.4094-2.9413$

C $-3.9129 \quad 1.5674-2.5309$

C -3.7273 $0.6349-1.5108$

$\mathrm{H}-4.88641 .6378-3.0108$

H -3.0297 $3.1339-3.7351$

H -3.9009 -2.76852 .1637$

H -1.6042 -3.0223 3.1096

$\mathrm{H}-4.3169-1.16160 .3638$

H $3.8598-2.3954-2.7338$

H $1.4926-2.5703-3.4440$

H 4.19511 .74983 .4786

H 1.89152 .40784 .1088

H -4.5660 $0.0045-1.2341$

H $4.4827-1.0718-0.7272$

H $4.60600 .4068 \quad 1.4283$

H -0.0433 1.74532 .6911

H $0.2452-1.62982 .2236$

H - $0.2934-1.3938-2.1717$

H -0.7957 $2.9220-2.5864$

Degenerate rearrangement of

diindeno[1,2,3,4- defg; $1^{\prime}, 2^{\prime}, 3^{\prime}, 4^{\prime}$ -

mnop]chrysene (Scheme 5)

\section{Hydrogen atom adduct to diindenochrysene}

SCF energy -998.558558618 a.u.

Zero-point correction 0.30149 a.u.

C $-0.1145 \quad 2.65320 .7538$

C $-0.11452 .6532-0.7538$

C $-0.6388 \quad 1.4137-1.1732$

C $-0.6388 \quad 1.4137 \quad 1.1732$

C $-0.0881-2.6675-0.7528$

C $-0.0881-2.66750 .7528$

C $-0.5528-1.3721 \quad 1.1485$

C $-0.9323-0.67250 .0000$

C $-0.5528-1.3721-1.1485$

C $-1.2725 \quad 0.73520 .0000$

C $-0.3008 \quad 0.7638 \quad 2.3734$ 
C 0.36531 .57843 .3160

C 0.72642 .89382 .9959

C $0.54893 .4311 \quad 1.7078$

C $0.54893 .4311-1.7078$

C $0.72642 .8938-2.9959$

C $0.3653 \quad 1.5784-3.3160$

C $-0.3008 \quad 0.7638-2.3734$

C $0.4299-3.4702 \quad 1.7619$

C $0.5203-2.93253 .0698$

C $0.2017-1.59913 .3821$

C $-0.3210-0.74412 .3941$

C $-0.3210-0.7441-2.3941$

C $0.2017-1.5991-3.3821$

C $0.5203-2.9325-3.0698$

C $0.4299-3.4702-1.7619$

H $0.9277-3.5616-3.8573$

H $0.4235-1.2305-4.3805$

H $0.9277-3.5616 \quad 3.8573$

H $0.4235-1.23054 .3805$

H $0.8115-4.47001 .5692$

H $1.24753 .4870-3.7433$

H $0.67361 .1690-4.2746$

H 1.24753 .48703 .7433

H 0.67361 .16904 .2746

H $0.8115-4.4700-1.5692$

H $0.98224 .3967-1.4597$

H $0.98224 .3967 \quad 1.4597$

H -2.3689 $0.9007 \quad 0.0000$

\section{Transition state}

SCF energy -998.45950827738 a.u. Zero-point correction 0.29773 a.u. Imaginary frequency $-518.23 \mathrm{~cm}^{-1}$

C $2.5724-0.7670-0.0887$

C $2.44410 .7156-0.1335$

C $1.0856 \quad 1.0493 \quad 0.0711$

C $1.3225-1.2514 \quad 0.2975$

C - 2.56600 .78980 .4309

C $-2.4024-0.6577 \quad 0.1544$

C $-1.0129-1.0467 \quad 0.1883$

C $-0.5343 \quad 0.1427 \quad 1.6054$

C - 1.39401 .24461 .0706

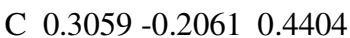

C $0.8385-2.5408 \quad 0.0898$

C $1.7972-3.5037-0.2752$

C $3.1180-3.0816-0.5066$

C $3.5180-1.7291-0.4671$

C $3.37351 .7584-0.2387$

C $2.93293 .0778-0.0770$

C $1.5987 \quad 3.3819 \quad 0.2367$

$\begin{array}{llll}C & 0.6502 & 2.3573 & 0.3527\end{array}$

C $-3.3526-1.6433-0.0751$

C $-2.9703-2.9936-0.2264$

C $-1.6315-3.3882-0.1270$

$\begin{array}{llll}C & -0.6290 & -2.4377 & 0.0887\end{array}$

C - 0.81752 .44930 .6176

C - 1.67463 .39770 .0340

C $-3.0003 \quad 3.0530-0.2646$

C $-3.42201 .7192-0.1717$
H -3.6507 $3.7896-0.7294$

H - $1.28074 .3521-0.3060$

H $-3.7351-3.7433-0.4084$

H $-1.3687-4.4370-0.2446$

H $-4.4065-1.3773-0.1012$

H $3.65173 .8892-0.1577$

H 1.31114 .41590 .4107

H $3.8521-3.8267-0.8039$

H $1.5312-4.5447-0.4400$

H $-4.33991 .4025-0.6600$

H $4.42731 .5536-0.4096$

H $4.5234-1.4575-0.7779$

H $-0.5085-0.24302 .6168$

Central intermediate

SCF energy -998.470210781 a.u.

Zero-point correction 0.29970 a.u.

C 2.54850 .05620 .7991

C $2.41820 .0020-0.6745$

C 1.0496 0.1226-1.0129

C $1.2852 \quad 0.3787 \quad 1.2964$

C $-2.51050 .4853-0.7698$

C -2.35720 .19090 .6830$

C -1.01130 .04391 .0831$

C $-0.3937 \quad 1.7482-0.3679$

C $-1.3628 \quad 1.1047-1.3030$

$\begin{array}{llll}C & 0.2239 & 0.5284 & 0.2637\end{array}$

C $0.8218-0.02122 .5511$

C $1.7829-0.43683 .4836$

C $3.1221-0.54313 .0662$

C $3.5136-0.35941 .7280$

C $3.3643-0.1244-1.7008$

C $2.9378-0.0669-3.0307$

C $1.59360 .1699-3.3596$

C $0.6308 \quad 0.3165-2.3546$

C $-3.3481-0.0341 \quad 1.6458$

C $-2.9961-0.31842 .9743$

C - $1.6602-0.34523 .3861$

C $-0.6510-0.13232 .4374$

$\begin{array}{llll}C & -0.8346 & 0.5722 & -2.4984\end{array}$

C $-1.7036-0.0680-3.3935$

C $-3.0233-0.3241-2.9956$

C $-3.3932-0.1520-1.6542$

H $-3.7069-0.8163-3.6824$

H $-1.3329-0.4720-4.3324$

H -3.7826 -0.4854 3.7057

H - $1.4145-0.50694 .4326$

H $-4.3976 \quad 0.0343 \quad 1.3702$

H $3.6679-0.1679-3.8296$

H $1.3047 \quad 0.2686-4.4031$

H $3.8665-0.87573 .7854$

H $1.5065-0.74584 .4884$

H $-4.3049-0.6126-1.2824$

H $4.4199-0.2450-1.4706$

H $4.5264-0.61141 .4244$

H $0.17802 .6269-0.6568$
Carbene-like transition state for non-catalysed rearrangement of diindeno[1,2,3,4- defg; $1^{\prime}, 2^{\prime}, 3^{\prime}, 4^{\prime}$ mnop]chrysene

SCF energy -997.813307719 a.u. Zero-point correction 0.28666 a.u. Imaginary frequency $-250.29 \mathrm{~cm}^{-1}$

C $-0.1273 \quad 2.3619 \quad 1.2348$

C $-0.1486 \quad 1.2407 \quad 2.2027$

C $-0.00250 .0205 \quad 1.4945$

C $0.2038 \quad 1.80910 .0000$

C $0.4876-2.3357-1.2085$

C $0.2059-1.2130-2.1477$

C $-0.00240 .0205-1.4945$

C $1.6004-0.4701-0.0002$

C $0.9922-1.82510 .0000$

$\begin{array}{llll}C & 0.3627 & 0.3379 & 0.0000\end{array}$

C $-0.1272 \quad 2.3619-1.2348$

C $-0.55163 .6985-1.2355$

C $-0.7087 \quad 4.35370 .0000$

C $-0.5517 \quad 3.6985 \quad 1.2355$

C $-0.28641 .1898 \quad 3.5934$

C -0.2079 -0.04994 .2418$

C $0.0504-1.23533 .5408$

C $0.2059-1.21302 .1478$

C $0.0503-1.2354-3.5407$

C $-0.2079-0.0499-4.2417$

C $-0.2863 \quad 1.1898-3.5934$

C $-0.1485 \quad 1.2407-2.2027$

C $0.4876-2.3357 \quad 1.2085$

C $-0.0516-3.62851 .2143$

C $-0.2226-4.3094-0.0000$

C $-0.0515-3.6285-1.2143$

$\mathrm{H}-0.6254-5.3183-0.0001$

$\mathrm{H}-0.4242-4.06582 .1375$

H $-0.3137-0.0892-5.3227$

H -0.4314 $2.0984-4.1717$

H $0.1564-2.1712-4.0831$

H -0.3137 -0.08925 .3228$

H $0.1566-2.17114 .0832$

H - $1.04995 .3858-0.0000$

$\mathrm{H}-0.8218 \quad 4.2129-2.1541$

H $-0.4241-4.0657-2.1376$

$\mathrm{H}-0.43162 .09844 .1717$

H -0.8217 4.2129 2.1541

Potential transition state with $\mathbf{C}_{2 \mathrm{v}}$ symmetry for concerted rearrangement of diindenochrysene

SCF energy -997.691228974 a.u. Zero-point correction 0.28209 a.u. Imaginary frequencies -1440.95 and $-356.76 \mathrm{~cm}^{-1}$

C $-0.1273 \quad 2.3619 \quad 1.2348$

C $-0.1486 \quad 1.2407 \quad 2.2027$

C $-0.0025 \quad 0.0205 \quad 1.4945$

$\begin{array}{llll}C & 0.2038 & 1.8091 & 0.0000\end{array}$ 
C $0.4876-2.3357-1.2085$

C $0.2059-1.2130-2.1477$

C $-0.00240 .0205-1.4945$

C $1.6004-0.4701-0.0002$

C $0.9922-1.82510 .0000$

$\begin{array}{llll}C & 0.3627 & 0.3379 & 0.0000\end{array}$

C - $0.1272 \quad 2.3619-1.2348$

C $-0.55163 .6985-1.2355$

C $-0.7087 \quad 4.35370 .0000$

C $-0.5517 \quad 3.6985 \quad 1.2355$

C $-0.28641 .1898 \quad 3.5934$

C $-0.2079-0.0499 \quad 4.2418$

C $0.0504-1.23533 .5408$

C $0.2059-1.21302 .1478$

C $0.0503-1.2354-3.5407$

C $-0.2079-0.0499-4.2417$

C $-0.2863 \quad 1.1898-3.5934$

C $-0.1485 \quad 1.2407-2.2027$

C $0.4876-2.3357 \quad 1.2085$

C - $0.0516-3.6285 \quad 1.2143$

C $-0.2226-4.3094-0.0000$

C $-0.0515-3.6285-1.2143$

$\mathrm{H}-0.6254-5.3183-0.0001$

$\mathrm{H}-0.4242-4.06582 .1375$

H $-0.3137-0.0892-5.3227$

H -0.4314 2.0984 -4.1717

H $0.1564-2.1712-4.0831$

H -0.3137 -0.0892 5.3228

H $0.1566-2.17114 .0832$

H - $1.04995 .3858-0.0000$

H -0.8218 4.2129 -2.1541

H -0.4241 -4.0657 -2.1376

H -0.4316 2.0984 4.1717

H - $0.8217 \quad 4.21292 .1541$

Radical-Promoted Stone-Wales rearrangement of $\mathrm{C}_{60}$

$\mathbf{C}_{60} \mathbf{I}_{\mathrm{h}}$-symmetric global minimum SCF energy -2286.139940 a.u.

C $0.7273-2.60242 .3066$

C $1.1768-1.42563 .0339$

C $1.4256-3.03391 .1768$

C $2.3066-0.7273 \quad 2.6024$

C $2.3066 \quad 0.72732 .6024$

C $0.0000-0.69833 .4834$

$\begin{array}{llll}C & 0.0000 & 0.6983 & 3.4834\end{array}$

C 1.17681 .42563 .0339

C - $0.6983-3.48340 .0000$

C - $1.4256-3.03391 .1768$

C $0.6983-3.48340 .0000$

C $-0.7273-2.60242 .3066$

C - $1.1768-1.42563 .0339$

C - $2.6024-2.30660 .7273$

C -3.0339 -1.17681 .4256$

C $-2.3066-0.72732 .6024$

C $0.7273-2.6024-2.3066$

C $-0.7273-2.6024-2.3066$

C $1.4256-3.0339-1.1768$
C - $1.4256-3.0339-1.1768$

C - $2.6024-2.3066-0.7273$

C $-1.1768-1.4256-3.0339$

C $-2.3066-0.7273-2.6024$

C $-3.0339-1.1768-1.4256$

C $3.0339-1.1768-1.4256$

C $2.3066-0.7273-2.6024$

C $2.6024-2.3066-0.7273$

C $1.1768-1.4256-3.0339$

C $0.0000-0.6983-3.4834$

C $2.30660 .7273-2.6024$

C $1.17681 .4256-3.0339$

C $0.00000 .6983-3.4834$

C $3.0339-1.17681 .4256$

C 3.48340 .00000 .6983

C $2.6024-2.30660 .7273$

C $3.48340 .0000-0.6983$

C $3.03391 .1768-1.4256$

C 3.03391 .17681 .4256

C 2.60242 .30660 .7273

C $2.60242 .3066-0.7273$

C $-0.72732 .6024-2.3066$

C $0.72732 .6024-2.3066$

C $1.42563 .0339-1.1768$

C - $1.42563 .0339-1.1768$

C $-3.03391 .1768-1.4256$

C $-2.30660 .7273-2.6024$

C - $1.1768 \quad 1.4256-3.0339$

C $-2.60242 .3066-0.7273$

C -3.0339 1.17681 .4256

C $-3.48340 .0000 \quad 0.6983$

C $-3.48340 .0000-0.6983$

C -2.60242 .30660 .7273$

C -0.72732 .60242 .3066$

C - $1.1768 \quad 1.42563 .0339$

C -2.3066 0.72732 .6024

C - 1.42563 .03391 .1768

C 0.69833 .48340 .0000

C 1.42563 .03391 .1768

C 0.72732 .60242 .3066

C -0.69833 .48340 .0000$

$\mathrm{C}_{60}$ Stone-Wales isomer $\left(\mathrm{C}_{2 \mathrm{v}}\right.$ symmetry)

SCF Energy -2286.069338 a.u.

C $2.26690 .7385 \quad 2.3334$

C 1.20101 .41943 .0302

C $2.8367 \quad 1.4304 \quad 1.2222$

C 0.73752 .66182 .5847

C $-0.7375 \quad 2.6618 \quad 2.5847$

C $0.0000-0.67703 .4570$

C $0.0000 \quad 0.67703 .4570$

C -1.2010 1.41943 .0302

C $3.3430-0.69190 .0701$

C $2.8367-1.43041 .2222$

C $3.3430 \quad 0.69190 .0701$

C $2.2669-0.73852 .3334$

C $1.2010-1.41943 .0302$
C $2.2675-2.66380 .7212$

C $1.1850-3.26231 .3823$

C $0.7375-2.66182 .5847$

C $2.58470 .7268-2.2805$

C $2.5847-0.7268-2.2805$

C $2.9867 \quad 1.4219-1.1396$

C $2.9867-1.4219-1.1396$

C $2.2905-2.6258-0.7335$

C $1.4241-1.1787-3.0308$

C $0.7292-2.3177-2.6180$

C $1.1810-3.0699-1.4609$

C $1.18103 .0699-1.4609$

C $0.7292 \quad 2.3177-2.6180$

C $2.29052 .6258-0.7335$

C $1.42411 .1787-3.0308$

C $0.69830 .0000-3.4819$

C $-0.7292 \quad 2.3177-2.6180$

C - $1.4241 \quad 1.1787-3.0308$

C $-0.69830 .0000-3.4819$

C 1.18503 .26231 .3823

C 0.00003 .68150 .6232

C 2.26752 .66380 .7212

C $0.00003 .5687-0.7598$

C - $1.18103 .0699-1.4609$

C $-1.1850 \quad 3.2623 \quad 1.3823$

C - $2.2675 \quad 2.6638 \quad 0.7212$

C - $2.29052 .6258-0.7335$

C - $2.5847-0.7268-2.2805$

C $-2.58470 .7268-2.2805$

C - $2.98671 .4219-1.1396$

C -2.9867 -1.4219-1.1396

C - $1.1810-3.0699-1.4609$

C $-0.7292-2.3177-2.6180$

C - $1.4241-1.1787-3.0308$

C - $2.2905-2.6258-0.7335$

C $-1.1850-3.2623 \quad 1.3823$

C $0.0000-3.68150 .6232$

C $0.0000-3.5687-0.7598$

C - $2.2675-2.66380 .7212$

C -2.2669 $-0.7385 \quad 2.3334$

C - $1.2010-1.41943 .0302$

C - $0.7375-2.66182 .5847$

C - $2.8367-1.4304 \quad 1.2222$

$\begin{array}{llll}C-3.3430 & 0.6919 & 0.0701\end{array}$

C - $2.8367 \quad 1.4304 \quad 1.2222$

C -2.2669 $0.7385 \quad 2.3334$

C -3.3430 -0.6919 0.0701

$\mathrm{C}_{60} \mathrm{H}$ radical adduct to groundstate C60

SCF energy -2286.707315 a.u.

C $2.58382 .2844-0.7292$

C $1.44673 .0311-1.1896$

C $3.03461 .1549-1.4282$

C $0.74212 .5875-2.2935$

C $-0.7332 \quad 2.5955-2.3018$

C 0.76773 .74080 .0000

C $-0.7361 \quad 3.50380 .0000$

C - $1.42673 .0207-1.1600$ 
C $3.4859-0.02000 .6978$

C 3.03461 .15491 .4282

C $3.4859-0.0200-0.6978$

C 2.58382 .28440 .7292

C 1.44673 .03111 .1896

C $2.3158 \quad 0.71002 .5991$

C $1.1797 \quad 1.41193 .0169$

C 0.74212 .58752 .2935

C $2.6024-2.3252-0.7280$

C $2.6024-2.32520 .7280$

C $3.0383-1.1976-1.4262$

C $3.0383-1.19761 .4262$

C $2.3116-0.74432 .6030$

C $1.4226-3.0470 \quad 1.1763$

C $0.7249-2.61032 .3056$

C $1.1769-1.43663 .0307$

C $1.1769-1.4366-3.0307$

C $0.7249-2.6103-2.3056$

C $2.3116-0.7443-2.6030$

C $1.4226-3.0470-1.1763$

C $0.6943-3.49450 .0000$

C $-0.7326-2.6072-2.3057$

C - $1.4305-3.0408-1.1765$

C $-0.7035-3.48880 .0000$

C $1.1797 \quad 1.4119-3.0169$

C $0.00040 .6902-3.4651$

C $2.3158 \quad 0.7100-2.5991$

C $-0.0017-0.7041-3.4761$

C - $1.1808-1.4326-3.0324$

C $-1.1773 \quad 1.4239-3.0195$

C -2.3156 $0.7176-2.6003$

C $-2.3122-0.7323-2.6017$

C - $2.6096-2.31440 .7281$

C - $2.6096-2.3144-0.7281$

C - $3.0432-1.1874-1.4261$

C -3.0432 -1.18741 .4261$

C $-1.1808-1.43263 .0324$

C $-0.7326-2.60722 .3057$

C - $1.4305-3.0408 \quad 1.1765$

C $-2.3122-0.73232 .6017$

C $-1.1773 \quad 1.42393 .0195$

C 0.00040 .69023 .4651

C -0.0017 -0.70413 .4761$

C -2.3156 0.71762 .6003

C - $2.6126 \quad 2.2877 \quad 0.7238$

C $-1.42673 .0207 \quad 1.1600$

C -0.73322 .59552 .3018$

C $-3.0452 \quad 1.16391 .4250$

C $-3.4975-0.0125-0.6978$

C - $3.04521 .1639-1.4250$

C $-2.61262 .2877-0.7238$

C -3.4975 $-0.0125 \quad 0.6978$

H $0.9981 \quad 4.8250 \quad 0.0000$

First transition state for rearrangement of $\mathrm{C}_{60} \mathrm{H}$ to the intermediate.

SCF energy -2286.524286 a.u.

C $2.29890 .6428-2.1969$
C $2.77711 .0887-0.9145$

C $1.27561 .4127-2.7909$

C $2.58302 .4024-0.5370$

C 2.64062 .52600 .9001

C $4.2404-0.5167-0.6427$

$\begin{array}{llll}C & 3.3409 & 0.2167 & 0.3504\end{array}$

C 3.11491 .35111 .4638

C $0.0969-0.6692-3.3691$

C $1.2513-1.4377-2.9220$

C $0.1193 \quad 0.7210-3.3491$

C $2.4002-0.7884-2.4155$

C $3.2675-1.4039-1.4345$

C $0.7577-2.6319-2.2746$

C $1.4432-3.0991-1.1471$

C $2.6557-2.4452-0.7461$

C - $2.21550 .7903-2.5578$

C - $2.2337-0.6641-2.5602$

C $-1.0675 \quad 1.4725-2.9614$

C $-1.1078-1.3796-2.9754$

C $-0.6985-2.5840-2.2708$

C -2.9814 -1.1036 -1.3942

C - $2.5694-2.2394-0.6923$

C $-1.4075-2.9889-1.1350$

C $-1.35853 .1140-1.1252$

C $-2.53702 .3833-0.6937$

C - $0.63562 .6557-2.2323$

C $-2.9602 \quad 1.2521-1.3951$

C $-3.42700 .0785-0.6726$

C $-2.55842 .3883 \quad 0.7641$

C $-2.99761 .2576 \quad 1.4559$

C -3.44260 .08090 .7238$

C $1.47653 .1487-1.0638$

C 0.73583 .63620 .0963

C $0.80862 .6377-2.1760$

C $-0.6593 \quad 3.59000 .0635$

C $-1.39903 .1269 \quad 1.2323$

C $1.45773 .2317 \quad 1.3122$

C 0.74062 .74062 .4124

C $-0.71392 .7051 \quad 2.3770$

C -2.2868 -0.64662 .6379$

C -2.2817 0.80812 .6382

C - $1.1545 \quad 1.50833 .0773$

C - $1.1617-1.34583 .0750$

C $-1.4200-2.9741 \quad 1.2251$

C $-2.5805-2.23340 .7651$

C -3.0080 -1.0973 1.4568

C $-0.7206-2.5323 \quad 2.3515$

C $1.4282-3.0023 \quad 1.2170$

C $0.6993-3.48500 .0510$

C -0.6944 -3.4499 0.0532

C $0.7281-2.53172 .3361$

C $2.2224-0.59372 .4418$

C $2.7660-0.98901 .1912$

C $2.5646-2.26790 .7131$

C $1.1773-1.33883 .0063$

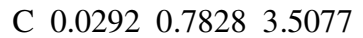

C 1.20441 .53713 .0696

C 2.31960 .83972 .5517

C $0.0203-0.61243 .5077$

H $5.3197-0.5590-0.7261$

\section{Intermediate for rearrangement of $\mathrm{C}_{60} \mathrm{H}$}

SCF energy -2286.528577 a.u.

C $2.29300 .6722-2.1999$

C $2.77041 .1281-0.9258$

C $1.25861 .4339-2.7926$

C $2.55612 .4334-0.5494$

C 2.62812 .55430 .8890

C $4.2665-0.5837-0.6061$

$\begin{array}{llll}\text { C } 3.3479 & 0.2603 & 0.2919\end{array}$

C 3.11781 .38131 .4428

C $0.0906-0.6574-3.3606$

C $1.2499-1.4198-2.9137$

C $0.10390 .7325-3.3434$

C $2.3955-0.7626-2.4042$

C $3.2697-1.3929-1.4423$

C $0.7619-2.6253-2.2821$

C $1.4543-3.1058-1.1636$

C $2.6679-2.4555-0.7721$

C - $2.23040 .7856-2.5463$

C -2.2401 -0.6687 -2.5506

C - $1.08841 .4756-2.9541$

C - $1.1105-1.3763-2.9691$

C $-0.6938-2.5815-2.2712$

C - $2.9818-1.1145-1.3824$

C $-2.5607-2.2495-0.6843$

C - $1.3979-2.9934-1.1338$

C $-1.38333 .1139-1.1158$

C - $2.55482 .3744-0.6791$

C $-0.66292 .6619-2.2275$

C -2.9733 $1.2413-1.3799$

C -3.4305 $0.0640-0.6569$

C - $2.5692 \quad 2.37760 .7792$

C $-2.9970 \quad 1.2428 \quad 1.4717$

C -3.43920.0646 0.7400

C $1.44783 .1769-1.0694$

C 0.71083 .65370 .0970

C $0.78252 .6558-2.1788$

C $-0.6832 \quad 3.5951 \quad 0.0712$

C - $1.4128 \quad 3.1233 \quad 1.2431$

C $1.44323 .2507 \quad 1.3088$

C 0.73642 .75102 .4117

C -0.71862 .70502 .3842$

C - $2.2680-0.65772 .6473$

C -2.2714 0.79652 .6489

C - $1.1461 \quad 1.5031 \quad 3.0834$

C - $1.1365-1.35103 .0772$

C - $1.3980-2.98031 .2269$

C $-2.5648-2.2450 \quad 0.7738$

C - $2.9939-1.11191 .4695$

C -0.6947 -2.5344 2.3499

C $1.4465-2.99401 .2008$

C $0.7154-3.48710 .0412$

C $-0.6780-3.4540 \quad 0.0508$

C $0.7539-2.52452 .3238$

C $2.2362-0.57332 .4097$

C $2.7581-0.9617 \quad 1.1479$

C $2.5720-2.25160 .6837$ 
C $1.2037-1.32882 .9879$

$\begin{array}{llll}\text { C } & 0.0458 & 0.7848 & 3.5039\end{array}$

C $1.2137 \quad 1.54803 .0597$

C 2.33040 .85922 .5293

C $0.0460-0.61023 .5007$

H $5.1728-1.0696-0.2530$

Second transition state for rearrangement of the intermediate to the $\mathbf{C}_{60} \mathbf{H}$ isomer.

SCF energy -2286.506917 a.u.

C $2.30450 .7181-2.2356$

C $2.83691 .2238-1.0104$

C $1.24321 .4624-2.8127$

C $2.55722 .5065-0.5978$

C 2.61642 .56790 .8511

C $4.2237-0.6124-0.5431$

C 3.44940 .41340 .1515

C 3.11641 .37731 .3704

C $0.0876-0.6424-3.3438$

C $1.2470-1.3932-2.8832$

C $0.09060 .7477-3.3393$

C $2.3827-0.7267-2.3704$

C $3.2601-1.3703-1.4103$

C $0.7649-2.6092-2.2644$

C $1.4581-3.1023-1.1522$

C $2.6825-2.4700-0.7503$

C - $2.24340 .7871-2.5429$

C $-2.2461-0.6676-2.5460$

C $-1.10651 .4831-2.9535$

C $-1.1111-1.3683-2.9593$

C $-0.6901-2.5716-2.2616$

C $-2.9900-1.1175-1.3810$

C $-2.5670-2.2525-0.6855$

C - $1.4046-2.9967-1.1358$

C - $1.40473 .1198-1.1179$

C - $2.56872 .3734-0.6759$

C - $0.68562 .6746-2.2338$

C $-2.98651 .2388-1.3765$

C $-3.43870 .0602-0.6533$

C - $2.5782 \quad 2.3737 \quad 0.7825$

C -3.0006 $1.2362 \quad 1.4746$

C -3.4453 $0.0597 \quad 0.7438$

C $1.4273 \quad 3.2198-1.0943$

C $0.69043 .6708 \quad 0.0841$

C $0.76112 .6844-2.1999$

C $-0.7018 \quad 3.6012 \quad 0.0677$

C $-1.4205 \quad 3.1183 \quad 1.2415$

C $1.43143 .2491 \quad 1.2832$

C $0.73802 .7281 \quad 2.3797$

C $-0.7143 \quad 2.69002 .3718$

C -2.2671 -0.66712 .6444$

C -2.2668 0.78702 .6469

C $-1.1349 \quad 1.4885 \quad 3.0713$

C - $1.1358-1.36143 .0704$

C $-1.4027-2.98681 .2233$

C - $2.5694-2.2507 \quad 0.7728$

C -2.9972 -1.1184 1.4698

C - $0.6972-2.54762 .3489$
C $1.4338-3.04651 .2096$

C $0.7059-3.50260 .0400$

C $-0.6870-3.4604 \quad 0.0455$

C $0.7518-2.55472 .3326$

C $2.2758-0.62222 .4557$

C $2.8668-1.14151 .3026$

C $2.5976-2.34080 .7185$

C $1.2074-1.35623 .0068$

C 0.06080 .76643 .4780

C $1.2277 \quad 1.51803 .0071$

$\begin{array}{lll}\text { C } 2.3432 & 0.8282 & 2.4715\end{array}$

C $0.0494-0.62673 .4917$

H $4.9933-1.1933-0.0413$

Rearranged "Stone-Wales-like"

Isomer of $\mathrm{C}_{60} \mathrm{H}$ radical

SCF energy -2286.648631 a.u.

C $2.32410 .7343-2.2556$

C 3.0208 1.4185-1.1817

C $1.2199 \quad 1.4249-2.8287$

C $2.57442 .6818-0.7334$

C 2.57442 .68180 .7334

C $3.7054-0.73920 .0000$

C 3.44330 .70840 .0000

C $3.0208 \quad 1.41851 .1817$

C $0.0610-0.6943-3.3354$

C $1.2060-1.4336-2.8255$

$\begin{array}{llll}C & 0.0678 & 0.6905 & -3.3327\end{array}$

C $2.3192-0.7472-2.2504$

C $3.0253-1.4384-1.2064$

C $0.7002-2.6717-2.2660$

C $1.3584-3.2671-1.1852$

C $2.5602-2.6573-0.7384$

C $-2.2845 \quad 0.7317-2.5841$

C $-2.2898-0.7221-2.5847$

C - $1.1407 \quad 1.4236-2.9828$

C $-1.1513-1.4208-2.9866$

C $-0.7514-2.6294-2.2918$

C - $3.0425-1.1717-1.4248$

C $-2.6347-2.3126-0.7297$

C -1.4812 -3.0699-1.1806

C - $1.46903 .0797-1.1810$

C -2.6217 $2.3256-0.7309$

C $-0.73492 .6303-2.2886$

C -3.0363 $1.1864-1.4253$

C -3.4873 $0.0091-0.6988$

C -2.6217 2.32560 .7309

C $-3.0363 \quad 1.18641 .4253$

C -3.48730 .00910 .6988$

C $1.37113 .2725-1.1833$

C 0.61013 .69720 .0000

C $0.71312 .6688-2.2636$

C -0.77213 .58220 .0000$

C - $1.46903 .0797 \quad 1.1810$

C 1.37113 .27251 .1833

C 0.71312 .66882 .2636

C $-0.73492 .6303 \quad 2.2886$

C - $2.2898-0.7221 \quad 2.5847$

C -2.2845 $0.7317 \quad 2.5841$
C - $1.1407 \quad 1.42362 .9828$

C - $1.1513-1.42082 .9866$

C - $1.4812-3.06991 .1806$

C - $2.6347-2.31260 .7297$

C - $3.0425-1.1717 \quad 1.4248$

C $-0.7514-2.62942 .2918$

C $1.3584-3.26711 .1852$

C $0.6019-3.68260 .0000$

C $-0.7838-3.57040 .0000$

C $0.7002-2.67172 .2660$

C $2.3192-0.74722 .2504$

C $3.0253-1.43841 .2064$

C $2.5602-2.65730 .7384$

C $1.2060-1.43362 .8255$

$\begin{array}{llll}C & 0.0678 & 0.6905 & 3.3327\end{array}$

C 1.21991 .42492 .8287

C $2.32410 .7343 \quad 2.2556$

C $0.0610-0.69433 .3354$

H $4.7929-0.93250 .0000$ 